\title{
A Design of CMOS Broadband Amplifier With High-Q Active Inductor
}

\author{
Jhy-Neng Yang ${ }^{(1)(2)}$, Yi-Chang Cheng ${ }^{(2)}$, Chen-Yi Lee ${ }^{(1)}$ \\ (1) Department of Electronics Engineering and Institute of Electronics \\ National Chiao Tung University \\ Engineering $4^{\text {th }}$ Building, 1001 Ta-Hsueh Road, Hsing-Chu 300, Taiwan. \\ (2) Department of Electronics Engineering Minghsin University of Science Technology \\ 1. Hsin-Hsing Rd., Hsin-Fong, Hsin-Chu 304, Taiwan. \\ Tel: 886-3-5593142EXT3143, Fax: 886-3-5591402,Email:yjn@must.edu.tw
}

\begin{abstract}
A CMOS broadband amplifier with high- $Q$ active inductor using $0.25 \mathrm{um}$ CMOS process is presented. In this broadband amplifier, the compact high- $Q$ active inductor is connected to the common-gate configuration to improve the performance of the high power gain, wide bandwidth, low power consumption and simple matching characteristics. Not using any passive inductor components is to be reduced the area of chip and the complexity. Advance Design System (ADS) simulator has been performed to verify the performance of the designed broadband amplifier. It has been shown that the amplifier has a 20dB(S21) power gain in $-3 d B$ bandwidth, S11 of $-17 d B, S 22$ of $-21 \mathrm{~dB}$ and noise figure (NF) of $8 \mathrm{~dB}$, under $2.5 \mathrm{~V}$ power supply with $18 \mathrm{~mW}$ power consumption. ${ }^{*}$
\end{abstract}

\section{Introduction}

Motivated by the growing market of wireless communications system, instrumentations and optical communications much effort have been devoted to the implementation of components in CMOS technology. The broadband amplifier is the demanding block in broadband telecommunication system. The specifications of broadband amplifier must be satisfied simultaneously including, wide bandwidth, large power gain, good impedance matching, good linearity, low power consumption and low cost. Most of the published in these applications are implemented by using on chip passive spiral inductors to achieve good matching and power gain [1][2]. Although, the quality factor of an integrated passive inductor is

\footnotetext{
* This work was supported by the National Science Council of Taiwan, R. O. C., under Grant NSC 91-2626-E-159-004.
}

normally low. A high quality factor often requires additional processing steps. Nevertheless, by applying techniques such as the ones proposed in [3] it is possible to compensate the quality factor of these inductors, involving extra cost. Moreover, the inductor value is dependent on the size of the inductor [4]. The chip area occupied by an integrated passive inductor is usually large compared to other components. The above difficulties can be overcome by using an active inductor. Most of the proposed active inductor circuits using low noise amplifier or band-pass filter have been employed [5][6][7], but it is few used in broadband amplifiers. It will be improved the performance of broadband amplifiers using active inductor. An active inductor is usually implemented from a gyrator where two transconductors are connected in back-to-back configuration [8]. However, the active inductor circuits need to use active devices in integrated circuit implementation. The nonideal characteristics of active devices such as DC bias will limit the performance of the active inductor circuits such as the Q-value [9]. The limitations of $Q$ of the active inductor will reduce the performance of the broadband amplifier. Therefore, a broadband amplifier using high Q-value active inductor is proposed. In this paper, we carry out a description of a broadband amplifier how through using high Q-value active inductor design to improve the performance of the broadband amplifier. The design of the high Q-value active inductor is given in section 2. The design of the broadband amplifier is given in section 3. Simulation results of the proposed broadband amplifier are shown in 4 . Finally, the conclusion is given in section $\mathbf{5}$.

\section{High-Q active inductor design}




\subsection{A Simple Cascode Active Inductor}

An often-used way for making active inductors is through the combination of a gyrator where two transconductors are connected in back-to-back configuration and a capacitor [8]. The simple cascode active inductor shown in Fig.1, exploit the parasitic within the devices. In this circuit, transistor $\mathrm{M}_{1}$ is used to converter the input voltage $\mathrm{V}_{\mathrm{s}}$, to be a current for charging the integrating capacitor $C_{g s l}$, whereas $\mathrm{M}_{2}$ is used to converter the voltage across $C_{g s 2}$ to the input current $\mathrm{I}_{\mathrm{s}}$.

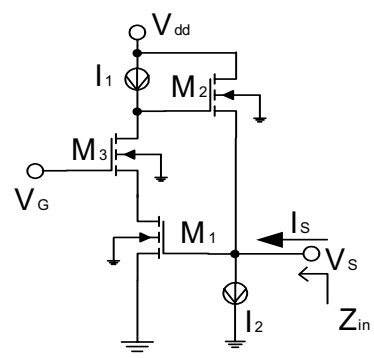

Fig. 1 A simple cascode active inductor

In this configuration, the gain boosting is utilization around the cascode common-source amplifier comprising of $\mathrm{M}_{3}, \mathrm{M}_{1}$ and $\mathrm{I}_{1}$ to reduce the series resistance. Hence, gain-boosting techniques have been applied to $\mathrm{M}_{3}$ in order for increasing its voltage gain, for cascode configuration. However, in Fig. 1, the ideal current source $I_{1}$ and $I_{2}$ will be replaced with the practical active device of MOSFET or current mirror circuit in the integrated circuit fabrication, which shown in Fig. 2.

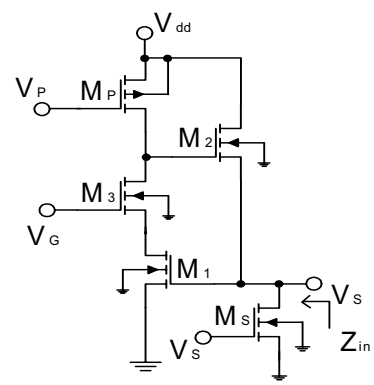

Fig. 2 The practical simple active inductor

Owing to the drain to source nonideal conductance of the active devices $M_{P}$ and $M_{S}$ cause the internal loss increasing of active inductor. The performance of the active inductor will be disrupted such as the Q-value. The performance of S11 of the practical simple active inductor is shown in Fig. 3. According to the Fig. 3, besides the Q-value is reduced, the operating frequency and inductance are also reduced.

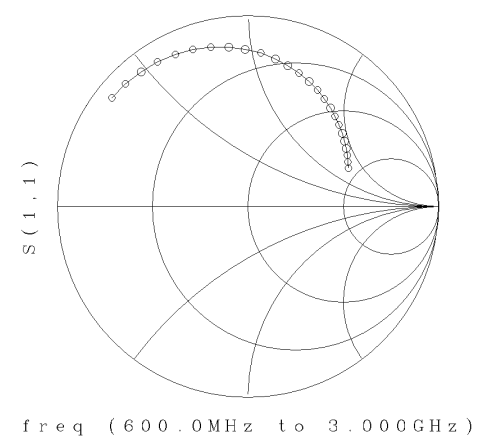

Fig. 3 The performance S11 of the practical active inductor

\subsection{A proposed high $Q$ active inductor}

The performance of the practical cascode active inductor in Fig. 2 can be improved by using the RC feedback cascode network to compensate the internal loss owing to the nonideal conductance of the active devices $\mathrm{M}_{\mathrm{P}}, \mathrm{M}_{\mathrm{S}}$ and other active device nonideal internal loss, which shown in Fig. 4.

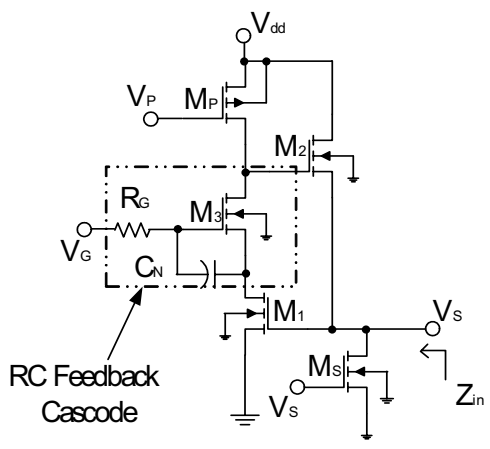

Fig. 4 The high $\mathrm{Q}$ active inductor

The high $\mathrm{Q}$ active inductor network is using $\mathrm{RC}$ feedback cascode positive feedback formed by $M_{3}, R_{G}$ 
and $\mathrm{C}_{\mathrm{N}}$ to compensate the internal loss of the active devices $\mathrm{M}_{\mathrm{P}}$ and $\mathrm{M}_{\mathrm{S}}$ and other internal loss factors. In this RC feedback cascode network, which is using the gain boosting of cascode configuration and generating negative impedance to compensate the conductance of active devices. $\mathrm{M}_{3}$ transistor is used to form the cascode with $\mathrm{M}_{1}$ to reduce the conductance of $\mathrm{M}_{1}$. $\mathrm{R}_{\mathrm{G}}$ and $\mathrm{C}_{\mathrm{N}}$ conduct with the M1 to form the negative impedance, which compensates the conductance of transistor $\mathrm{Mp}$ and $\mathrm{M}_{\mathrm{S}}$. Therefore, the performance of the active inductor can be improve by the RC feedback cascode configuration. The performance of Q-enhancement active inductor is shown in Fig. 5. From the Fig. 5, it is seen that the Q-value and operating frequency are increased. Owing to the conductance loss, the performance of active inductor can be improved using RC feedback cascode configuration.

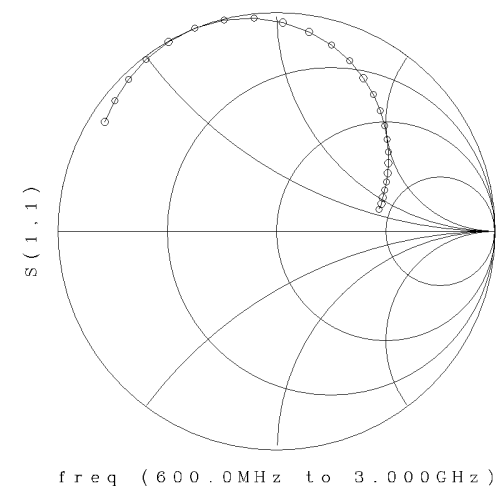

Fig. 5 The performance of Q-enhancement active inductor

\section{The design of broadband amplifier using high- $Q$ active inductor}

A common gate configuration CMOS broadband amplifier using improve high-Q active inductor is shown in Fig. 6. In this circuit consists of three different stages, including common gate configuration, high $\mathrm{Q}$ active inductor and buffer. Transistors $\mathrm{M}_{\mathrm{S} 1}$ and $\mathrm{M}_{\mathrm{S} 2}$ comprise the input common gate amplifier stage. This common-gate configuration provides a simple 50 $\Omega$ input matching and higher linearity without source degeneration inductor. This common-gate approach also helps to increase the effective reverse isolation.
The high-Q active inductor is constructed by transistors $\mathrm{M}_{1} \sim \mathrm{M}_{3}, \mathrm{M}_{\mathrm{P}}, \mathrm{R}_{\mathrm{G}}$ and $\mathrm{C}_{\mathrm{N}}$. It forms as the load of the common gate configuration. The key factor to obtain high circuit gain is to increase the Q value of active inductor. Therefore, the high- $Q$ active inductor is using positive feedback to compensate the internal loss and cascode configuration to improve the series resistive loss so that the Q-value of the active inductor is increased and the gain of broadband amplifier can be increased. Transistor $M_{B}$ and $R_{B}$ construct the output buffer stage. This common drain configuration provides minimizing the loading effect

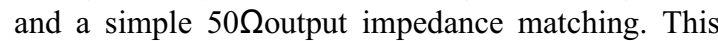
broadband amplifier is sensitive to the parasitic capacitance, and any following stage loading. Theses will be modified the overall circuit response by tuning the biases of $\mathrm{V}_{\mathrm{S} 1}, \mathrm{~V}_{\mathrm{S} 2}, \mathrm{~V}_{\mathrm{G}}$ and $\mathrm{V}_{\mathrm{P}}$. Therefore, it is easily to tune the variation due to the process or other factors. Capacitors $\mathrm{C}_{\mathrm{B} 1}$ and $\mathrm{C}_{\mathrm{B} 2}$ are used as a $\mathrm{DC}$ blocking capacitor of the input and output to isolate DC voltage of the previous stage and following stage.

Fig. 6 The broadband amplifier using high-Q active

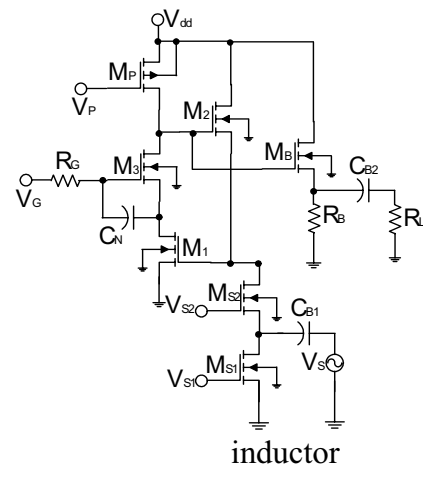

\section{Simulation results}

The complete broadband amplifier was simulated with parameters from a 0.25 -um CMOS process technology using ADS simulator. According to the circuit Fig.6, each transistor has the same as width and length. The minimum lengths and width are $0.24 \mathrm{um}$ and 40um respectively. The biasing values are shown as follows: $\mathrm{V}_{\mathrm{S} 1}=\mathrm{V}_{\mathrm{S} 2}=1 \mathrm{~V}, \mathrm{~V}_{\mathrm{G}}=1.7 \mathrm{~V}, \mathrm{~V}_{\mathrm{P}}=1.4 \mathrm{~V}$, and the normal supply voltage is $2.5 \mathrm{~V}$. In the Figs. 7 is shown the simulated results of $\mathrm{S} 21, \mathrm{~S} 11, \mathrm{~S} 12$ and noise figure (NF) respectively. According to the Fig. 7, the 
bandwidth of amplifier is about $1 \mathrm{GHz}$. It can be seen that $\mathrm{S} 21$ is $20 \mathrm{~dB}$ in the $-3 \mathrm{~dB}$ cutoff frequency and $\mathrm{S} 11$ and $\mathrm{S} 22$ are $-17 \mathrm{~dB}$ and $-21 \mathrm{~dB}$ respectively. It is obtained good performance using the improved high Q active inductor. In the Fig. 8, the noise figure (NF) is about $8 \mathrm{~dB}$ in the range of $0.1 \mathrm{GHz}$ to $3 \mathrm{GHz}$. The noise figure can be met the requirement in telecommunication system applications.

\section{Conclusion}

Previous works on broadband amplifier have relied on the use of integrated passive inductors as tuned elements. This paper presents the design of a CMOS broadband amplifier using a improved high-Q active inductor load. The proposed circuit was verified by ADS simulation, which demonstrated that the bandwidth and Q-value of the broadband amplifier and the active inductor have good performance. The performance, the power gain, noise figure and matching of the amplifier are found to be better those employed integrated passive inductor and other broadband amplifier. This work is available in most telecommunication system and optical applications.

\section{Reference}

[1] A. Rofougaran, J. Y-C. Chang, M. Rofougaran, A. A. Abidi, "A $1 \mathrm{GHz}$ CMOS RF Front-End IC for a Direct-Conversion Wireless Receiver," IEEE J. Solid-State Circuits, vol. 31, pp.880-889, July 1996.

[2] D. K. Shaffer and T. H. Lee, “ A 1.5GHz CMOS Low Noise Amplifier," IEEE J. Solid-State Circuits, vol. 32, pp.745-759, May 1997.

[3] J. Y. C. Chang, A. A. Abidi, and M. Gaitain, " Large Suspended Inductors On Silicon and Their use in a 2-um CMOS RF Amplifier," IEEE Electronics Device Lett., vol. 14, pp. 246-248, May 1993.

[4] H. M. Greenhouse, "Design of Planar Rectangular Microelectronic Inductors," IEEE Trans. Parts, Hybrids and Packaging, Vol. PHP-10, pp.101-109, June 1974.

[5] W. Zhuo, J. Pineda de Gyvez, E. Sanchez-Sinencio, "Programmable Low Noise Amplifier ith Active Inductor Load," IEEE International Symposium on Circuits and Systems, vol. 4, pp.365-368, 1998.

[6] U Yodprasit,. and J. Ngarmnil, " Q-Enhancement Technique for RF CMOS Active Inductor," IEEE ISCAS 2000 Circuits and systems, vol. 5, pp. 589-592.
[7] Y. Chang J. Choma, Jr, J. Wills, "A 900MHz Active Inductor LNA with A Band pass Filter," SSMSD '99', Symposium on Mixed-Signal Design, pp.33-36, 1999.

[8] A. Thanachaynont, and A. Payne, "VHF CMOS integrated active inductor," Electronics Lett., vol. 32, no. 11, pp. 999-1000, May 1996.

[9] Wu, Y., Ismail, M., and Olsson, H.: 'CMOS VHF/RF CCO based on active inductors', Electron. Lett., 2001, 37, (8), pp. 472-473

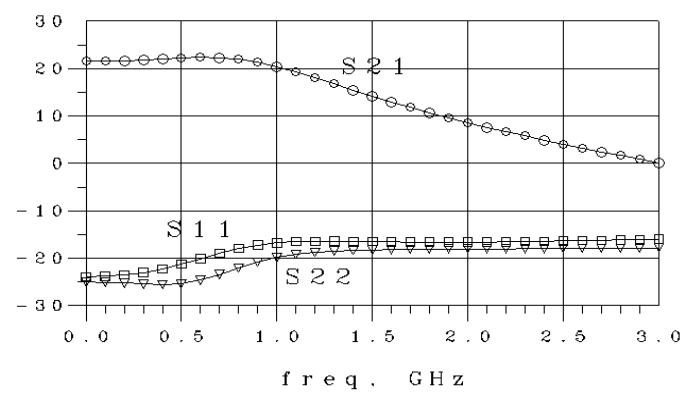

Fig. 7 The S21, S11 and S22 of the broadband amplifier

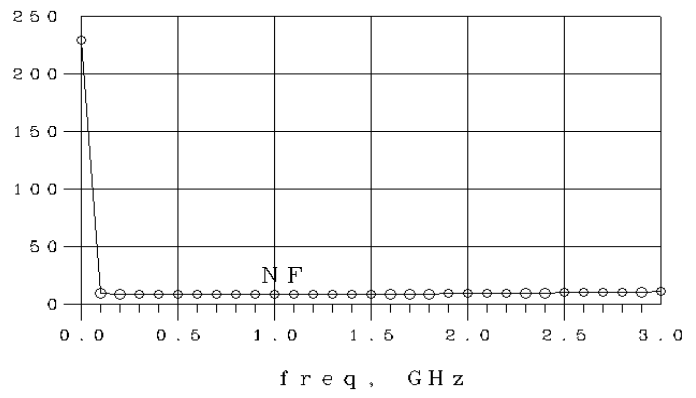

Fig. 8 The noise figure (NF) of the broadband amplifier 